\title{
DESEMPENHO OPERACIONAL DE SEMEADORA-ADUBADORA EM DIFERENTES MANEJOS DA COBERTURA E DA VELOCIDADE ${ }^{1}$
}

\author{
CARLOS E. A. FURLANI ${ }^{2}$, ÁlVARO PAVAN JÚNIOR ${ }^{3}$, AFONSO LOPES ${ }^{4}$, \\ ROUVERSON P. SILVA ${ }^{5}$, DANILO C.C. GROTTA ${ }^{3}$, JORGE W. CORTEZ ${ }^{3}$
}

\begin{abstract}
RESUMO: O presente trabalho teve como objetivo avaliar o desempenho de uma semeadoraadubadora no sistema plantio direto. Os fatores estudados foram três manejos das culturas de cobertura, selecionados em função do tamanho de fragmentos da vegetação, triturador de palhas (palha totalmente triturada), roçadora (palha parcialmente picada) e rolo-facas (palha acamada), combinados com três velocidades do conjunto trator-semeadora-adubadora, sendo 4,0; 5,0 e $6,0 \mathrm{~km} \mathrm{~h}^{-1}$. O delineamento experimental foi em blocos casualizados, em esquema fatorial $3 \times 3$, com nove tratamentos e oito repetições, totalizando 72 observações. Para comparar os tratamentos, avaliaram-se a capacidade de campo operacional, a força de tração e a potência na barra, o consumo horário e por área, e a patinagem dos rodados do trator. O desempenho da semeadoraadubadora não foi influenciado pelos três manejos na cultura de cobertura vegetal. O aumento da velocidade provocou diminuição da força de tração, sendo o inverso para a capacidade de campo operacional e a potência na barra. O consumo horário de combustível aumentou com a velocidade, enquanto o operacional diminuiu.
\end{abstract}

PALAVRAS-CHAVE: força de tração, consumo de combustível, plantio direto.

\section{OPERATIONAL PERFORMANCE OF SEEDER IN DIFFERENT FORWARD SPEED AND WINTER COVER CROP MANAGEMENT}

\begin{abstract}
The present work aimed to evaluate the seeder performance in the direct sowing system. The studied factors were three cover crop managements, chosen according to the size of the vegetation fragment, such as straw (straw totally triturated), weeder (straw partially chopped) and knife-rolls (straw practically entire), combined with three speeds of the seeder, being 4.0; 5.0 and $6.0 \mathrm{~km} \mathrm{~h}^{-1}$. The experimental outlining was carried out in casual blocks in factorial scheme $3 \mathrm{x}$ 3 , with nine treatments and eight repetitions, totalizing 72 observations. In the course of the experiment the following variants were evaluated: effective field capacity, force and power in the bar, hourly and area consumption of fuel and tractor's pulleys sliding. The data reached were tabulated and submitted to factorial variant analysis. When the value of the F test was significant at 0.05 of probability, the Tukey test was carried out to compare the averages. The three managements in the vegetal covering culture did not influence the seeder performance. The force decreased with the increasing of the forward speed, the opposite happened to the operational field capacity and the power in the bar. The hourly consumption of fuel increased with the speed, while the operational decreased.
\end{abstract}

KEYWORDS: force, fuel consumption, direct sowing.

\footnotetext{
${ }^{1}$ Extraído da Dissertação de Mestrado em Agronomia (Ciência do Solo) do segundo autor.

${ }^{2}$ Prof. Adjunto, Departamento de Engenharia Rural/UNESP - Jaboticabal, Via de Acesso Prof. Paulo Donato Castellane, s/n, furlani@fcav.unesp.br, Bolsista de Produtividade CNPq.

${ }^{3}$ Pós-Graduando, Departamento de Engenharia Rural/UNESP - Jaboticabal - SP.

${ }^{4}$ Prof. Adjunto, Departamento de Engenharia Rural/UNESP - Jaboticabal - SP.

${ }^{5}$ Prof. Dr., Departamento de Engenharia Rural/UNESP - Jaboticabal - SP. Bolsista de Produtividade CNPq.

Recebido pelo Conselho Editorial em: 20-10-2006

Aprovado pelo Conselho Editorial em: 25-3-2007
}

Eng. Agríc., Jaboticabal, v.27, n.2, p.456-462, maio/ago. 2007 


\section{INTRODUÇÃO}

O sistema plantio direto consiste em fazer a semeadura em solo não preparado previamente, diferenciando dos outros processos de semeadura pela menor intensidade de mobilização do solo e pela redução da freqüência de tráfego de máquinas sobre o terreno e, ainda, manter sobre a superfície do mesmo grande quantidade de massa vegetal.

A manutenção da cobertura vegetal sobre o solo representa diferenças fundamentais entre plantio direto e preparo convencional (aração seguida de duas ou mais passadas da grade niveladora), cujos principais efeitos são: controle da erosão, menor variação da temperatura e da umidade do solo, maior eficiência agronômica, melhoria na dinâmica da matéria orgânica e estrutura do solo.

MARQUES et al. (1999) não constataram diferença no requerimento de força de tração na operação de semeadura em função dos diferentes manejos da vegetação espontânea (plantas daninhas). $\mathrm{O}$ valor médio obtido foi de $8,5 \mathrm{kN}$ (quatro linhas de semeadura e mecanismo de deposição de adubo tipo disco duplo concêntrico), sendo 2,2 kN por linha. Esse valor supera a faixa de força requerida por linha de $1,1 \mathrm{kN}$ a $2,0 \mathrm{kN}$ (ASAE, 1996) e dos valores obtidos por BOLLER et al. (1992) de 0,8 a 1,5 kN por linha.

SIQUEIRA et al. (1997) obtiveram 13,2 kN de exigência de força de tração média com semeadora-adubadora de seis linhas para soja, dotada de haste parabólica com ponteira de $25 \mathrm{~mm}$ (2,2 $\mathrm{kN}$ por linha).

$\mathrm{Na}$ operação de semeadura, MAHL et al. (2004), utilizando semeadora de seis linhas espaçadas de $0,45 \mathrm{~m}$, em três velocidades $\left(4,4 ; 6,1 \mathrm{e} 8,1 \mathrm{~km} \mathrm{~h}^{-1}\right)$, verificaram que a variação da velocidade interferiu no desempenho do conjunto, ou seja, o aumento da velocidade da menor para a maior proporcionou aumento de $86 \%$ na capacidade operacional do conjunto e redução de $26 \%$ no consumo de combustível por área trabalhada.

O objetivo do presente trabalho foi avaliar o desempenho operacional de uma semeadoraadubadora de precisão, em três manejos de cobertura de inverno (rolo-faca, triturador de palhas e roçadora) combinado com três velocidades $\left(4,0 ; 5,0\right.$ e $\left.6,0 \mathrm{~km} \mathrm{~h}^{-1}\right)$, na semeadura da soja.

\section{MATERIAL E MÉTODOS}

O experimento foi conduzido em área do Laboratório de Máquinas e Mecanização Agrícola (LAMMA), Departamento de Engenharia Rural/UNESP/Jaboticabal - SP. O local do presente trabalho está localizado nas coordenadas geodésicas: $21^{\circ} 14^{\prime}$ latitude $\mathrm{S}$ e $48^{\circ} 16^{\prime}$ longitude $\mathrm{W}$, com altitude média de $559 \mathrm{~m}$ e clima Cwa (subtropical), de acordo com a classificação de Köeppen. O solo apresentava como cultura de inverno a mucuna-cinza e o feijão-guandu.

A semeadura foi realizada em Latossolo Vermelho eutrófico típico, A moderado, textura argilosa e relevo suave ondulado (EMBRAPA, 1999).

Foi utilizado um trator Valtra, modelo BM-100, 4x2 TDA, com potência de 73,6 kW $(100 \mathrm{cv})$ no motor. Apresentava massa de $5.400 \mathrm{~kg}$ (40\% dianteiro e 60\% traseiro), pneus dianteiros de 14.9 - $24 \mathrm{R} 1 \mathrm{com} 3,8 \mathrm{~m}$ de perímetro e pressão de inflagem de 18 psi $(124 \mathrm{kPa})$, e pneus traseiros de 23.1 - $26 \mathrm{R} 1 \mathrm{com} 4,9 \mathrm{~m}$ de perímetro e pressão de inflagem de 22 psi (152 kPa). A força média na barra de tração foi determinada por meio de célula de carga (marca SHIMIZU) acoplada entre a barra de tração do trator e o cabeçalho da semeadora-adubadora. Para a aferição da velocidade, foi utilizado um radar acoplado ao trator. O consumo de combustível foi determinado em unidade de volume (mL), por meio de protótipo construído por LOPES et al. (2003). Todos os equipamentos estavam ligados a um sistema de aquisição de dados.

A semeadora-adubadora de precisão utilizada foi da Marchesan, modelo Cop Suprema, com sete linhas de semeadura, dotada de disco de corte para palhada de 18 " $(45,7 \mathrm{~cm})$, haste sulcadora com as seguintes características: $2,7 \mathrm{~cm}$ de espessura da ponteira, 1,0 cm de espessura da haste, 
distância do disco de corte à haste de $12 \mathrm{~cm}$, relação entre a altura e o comprimento da ponteira (H/L) de 1,06 e ângulo de ataque de $20^{\circ}$, disco duplo desencontrado de $15 "(38,1 \mathrm{~cm})$ para deposição da semente, sendo o distribuidor pneumático. O depósito de adubo possui capacidade para $1.310 \mathrm{~kg}$ e o de semente de $200 \mathrm{~kg}$, tendo a semeadora-adubadora $2.070 \mathrm{~kg}$, trabalhando com $655 \mathrm{~kg}$ de adubo na operação de semeadura.

Para o manejo da cultura de inverno, utilizaram-se as máquinas e os implementos apresentados na Tabela 1.

TABELA 1. Máquinas e implementos utilizados para o manejo da cultura de inverno.

\begin{tabular}{lccl}
\hline Equipamento & Marca & Modelo & Características \\
\hline Triturador & Jumil & $\begin{array}{c}\text { Trimax } \\
2500\end{array}$ & Montado, largura de corte de 2,3 m. \\
\hline Roçadora & Bertanha & RU 1.6 & Arrasto, largura de corte de $1,6 \mathrm{~m}$. \\
\hline Rolo-faca & LAMMA & $\begin{array}{l}\text { Arrasto, largura de corte de } 1,6 \mathrm{~m} \text {, cilindro de } 1 \mathrm{~m} \mathrm{de} \\
\text { diâmetro, massa de } 1.360 \mathrm{~kg} \text { e distância entre facas de } 0,2 \mathrm{~m}\end{array}$ \\
\hline
\end{tabular}

O delineamento experimental foi conduzido em blocos inteiramente casualizados, em esquema fatorial $3 \times 3$, com nove tratamentos e oito repetições, totalizando 72 observações. Os tratamentos foram as combinações de três manejos: triturador de palhas, rolo-faca e roçadora, juntamente com três velocidades teóricas de deslocamento: $4 ; 5$ e $6 \mathrm{~km} \mathrm{~h}^{-1}$. A parcela experimental ocupava área de $2.100 \mathrm{~m}^{2}(25 \times 12 \mathrm{~m})$, sendo $15 \mathrm{~m}$ entre as parcelas para realizar manobras e trânsito de máquinas e implementos.

A força de tração foi obtida diretamente pela célula de carga, enquanto a potência na barra de tração foi determinada de forma indireta, utilizando-se da eq.(1).

$$
\mathrm{PB}=\frac{\mathrm{FT} \mathrm{v}}{1000}
$$

em que,

$\mathrm{PB}$ - potência na barra, $\mathrm{kW}$;

FT - força de tração na barra, kN;

$\mathrm{v}$ - velocidade real de deslocamento, $\mathrm{m} \mathrm{s}^{-1}$, e

1000 - constante de transformação.

A capacidade de campo operacional foi determinada com base na largura real de trabalho da semeadora-adubadora e da velocidade real de deslocamento do conjunto, proporcionada pelas três velocidades. A largura de trabalho média obtida foi de $3,15 \mathrm{~m}$.

Para o cálculo da capacidade de campo operacional, foi utilizada a eq.(2).

$$
\mathrm{Cco}=\frac{\mathrm{Lmr} \mathrm{v}}{10} \text { ef }
$$

em que,

Cco - capacidade de campo operacional, ha $\mathrm{h}^{-1}$;

$\mathrm{v}$ - velocidade real de deslocamento, $\mathrm{km} \mathrm{h}^{-1}$;

Lmr - largura real de trabalho da máquina, m;

ef - eficiência (75\%), segundo MIALHE (1996), e

10 - constante de transformação.

Os consumos de combustível horário e operacional foram determinados utilizando-se das eqs.(3) e (4).

$$
\mathrm{Ch} \mathrm{v}=\frac{\mathrm{C} 3,6}{\mathrm{t}}
$$


em que,

Ch v - consumo horário, $\mathrm{L} \mathrm{h}^{-1}$;

$\mathrm{C}$ - volume consumido, $\mathrm{mL}$;

$\mathrm{t}$ - tempo de percurso na parcela, s, e

3,6 - fator de conversão.

$$
\mathrm{Co}=\frac{\mathrm{Ch} \mathrm{v}}{\mathrm{Cco}}
$$

em que,

Co - consumo operacional, $\mathrm{L} \mathrm{ha}^{-1}$, e

Cco - capacidade de campo operacional, ha $\mathrm{h}^{-1}$.

A patinagem foi avaliada utilizando sensores geradores de pulsos do modelo GIDP $6012 \mathrm{v}$, localizados no centro de cada uma das rodas, os quais realizam conversão de movimentos rotativos ou deslocamentos lineares em pulsos elétricos, gerando 60 pulsos por volta dos rodados do trator. Registrou-se o valor integrado do número de pulsos das rodas no momento da operação de semeadura (com carga na barra de tração). A condição sem carga foi determinada com o trator operando sem nenhuma máquina ou implemento acoplado à barra (ASAE, 1996). Para o cálculo da patinagem, utilizou-se da eq.(5).

$$
\text { Patinagem }=\left(1-\frac{\text { NPC }}{\text { NPS }}\right) 100
$$

em que,

NPC - número de pulsos da roda, trator operando com carga na barra de tração, e

NPS - número de pulsos da roda, trator operando sem carga na barra de tração.

Os dados obtidos foram tabelados e submetidos à análise de variância, e quando o valor do teste $\mathrm{F}$ (Fisher) foi significativo a $5 \%$ de probabilidade, foi realizado o teste de Tukey para a comparação de médias (PIMENTEL GOMES, 1987).

\section{RESULTADOS E DISCUSSÃO}

A capacidade de campo operacional (Cco) é apresentada na Tabela 2. Os diferentes manejos realizados na cultura do milheto não influenciaram na variável $\mathrm{Cco}$; isso mostra que, com a superfície do solo consolidada, sistema plantio direto, a fragmentação das culturas de cobertura não foram empecilho para a eficiência de tração dos rodados do trator. A Cco da operação de semeadura foi influenciada pela velocidade de deslocamento do conjunto, sendo estatisticamente maior nas velocidades mais altas, com valores de 1,$49 ; 1,73$ e $2,11 \mathrm{ha} \mathrm{h}^{-1}$, respectivamente, para velocidades de 4,0; 5,0 e $6,0 \mathrm{~km} \mathrm{~h}^{-1}$. Os tratamentos das velocidades foram incrementados em $25 \%$, de 4,0 para $5,0 \mathrm{~km} \mathrm{~h}^{-1} ; 20 \%$ de 5,0 para $6,0 \mathrm{~km} \mathrm{~h}^{-1}$, e $50 \%$ de 4,0 para $6,0 \mathrm{~km} \mathrm{~h}^{-1}$, porém os incrementos de capacidade de campo operacional foram de 16; 22 e 42\%, nessa ordem, resultados semelhantes aos encontrados por KLEIN et al. (2002). Diversos autores verificaram o efeito da velocidade de deslocamento sobre o aumento da Cco, dentre os quais, JUSTINO (1998) e MAHL (2006).

Na Tabela 2, são apresentados valores de força de tração e potência na barra na operação de semeadura. A força de tração e a potência exigidas na barra de tração não apresentaram diferença estatística nos três manejos. Segundo MAHL (2006), a palhada na superfície do solo dificulta a aderência dos rodados do trator ao solo, podendo aumentar a patinagem dos mesmos, causando, por conseqüência, aumento significativo da demanda de força de tração e potência na barra. $\mathrm{O}$ aumento da força de tração na barra está relacionado com o possível aumento da profundidade de semeadura, que foi observado por algumas medições na área experimental. 
Na maior velocidade de deslocamento, observou-se diminuição na força de tração, resultados semelhantes aos de SILVA (2000), e opostos aos encontrados por MAHL (2006). Sabendo-se que a demanda de potência é a relação direta entre a força de tração e a velocidade, constatou-se, neste experimento, que a exigência de potência na barra de tração foi menor para a menor velocidade, mesmo com a força de tração maior. Esse fato está diretamente relacionado à velocidade de deslocamento do conjunto trator-semeadora-adubadora. MAHL (2006) comenta que, à medida que aumentou a velocidade de deslocamento, ocorreu aumento gradativo na demanda de potência. FURLANI (2005) apresentou valores médios de força de tração de $20 \mathrm{kN}$ e para potência de $30 \mathrm{~kW}$ com velocidade de semeadura de $5 \mathrm{~km} \mathrm{~h}^{1}$ em plantio direto.

TABELA 2. Síntese da análise de variância para força de tração, potência na barra e capacidade de campo operacional.

\begin{tabular}{|c|c|c|c|}
\hline Fatores & FT $(\mathrm{kN})$ & $\mathrm{PB}(\mathrm{kW})$ & $\operatorname{Cco}\left(\right.$ ha h $\left.^{-1}\right)$ \\
\hline \multicolumn{4}{|l|}{ Manejos } \\
\hline Rolo-faca & $19,1 \mathrm{a}$ & $26,2 \mathrm{a}$ & $1,4 \mathrm{a}$ \\
\hline Triturador de palhas & $20,0 \mathrm{a}$ & 25,9 a & $1,3 \mathrm{a}$ \\
\hline Roçadora & $19,7 \mathrm{a}$ & $26,9 \mathrm{a}$ & $1,4 \mathrm{a}$ \\
\hline \multicolumn{4}{|l|}{ Velocidade $\left(\mathrm{km} \mathrm{h}^{-1}\right)$} \\
\hline 4,0 & $19,6 \mathrm{a}$ & $22,5 \mathrm{~b}$ & $1,1 \mathrm{c}$ \\
\hline 5,0 & $20,8 \mathrm{a}$ & $27,8 \mathrm{a}$ & $1,4 \mathrm{~b}$ \\
\hline 6,0 & $17,4 \mathrm{~b}$ & $28,8 \mathrm{a}$ & $1,6 \mathrm{a}$ \\
\hline \multicolumn{4}{|l|}{ Teste F } \\
\hline Manejos (M) & $1,4^{\mathrm{NS}}$ & $1,0^{\mathrm{NS}}$ & $0,5^{\mathrm{NS}}$ \\
\hline Velocidade (V) & $9,0 * *$ & $16,6^{* *}$ & $139,6 * *$ \\
\hline $\mathrm{Mx} \mathrm{V}$ & $0,4^{\mathrm{NS}}$ & $0,4^{\mathrm{NS}}$ & $0,5^{\mathrm{NS}}$ \\
\hline C.V. (\%) - Manejos & 8,9 & 9,5 & 8,0 \\
\hline C.V. (\%) - Velocidades & 14,5 & 15,6 & 7,3 \\
\hline
\end{tabular}

Em cada coluna, para cada fator, médias seguidas de mesmas letras minúsculas não diferem entre si, pelo Teste de Tukey, a 5\% de probabilidade. ${ }^{\text {NS }}$ : não-significativo $(\mathrm{P} \geq 0,05)$; *: significativo $(\mathrm{P} \leq 0,05)$; C.V.: coeficiente de variação.

A patinagem dos rodados do trator apresentada na Tabela 3 indica que não há diferença para os manejos da cobertura vegetal, estando esses valores dentro da faixa adequada de patinagem estabelecida por MIALHE (1996).

Observou-se diminuição da patinagem com o aumento da velocidade, resultados opostos aos encontrados por MAHL (2006). O coeficiente de variação está em um patamar alto, porém inferiores aos encontrados por MAHL (2002); em grande parte da literatura, os valores de coeficientes de variação referentes à patinagem são bastante altos.

Os consumos de combustível horário $\left(\mathrm{L} \mathrm{h}^{-1}\right)$ e operacional $\left(\mathrm{L} \mathrm{ha}^{-1}\right)$ na operação de semeadura são apresentados na Tabela 3. Os diferentes manejos da cobertura vegetal não influenciaram nos consumos de combustível horário e operacional; isso é comprovado pelos resultados obtidos da Cco, patinagem dos rodados, força e potência na barra de tração. Com o aumento da velocidade de deslocamento, houve aumento no consumo de combustível horário e diminuição no consumo de combustível operacional, apresentando diferença significativa. $\mathrm{O}$ aumento do consumo de combustível horário é explicado pela maior exigência do trator na maior velocidade, para o mesmo tempo de operação. No caso do consumo de combustível operacional, mesmo com a maior exigência do trator na maior velocidade, isso é compensado pela maior rapidez em semear um hectare; sendo assim, esse consumo acaba sendo menor na maior velocidade.

De acordo com NAGAOKA \& NOMURA (2003), o consumo horário de combustível no plantio direto foi de $4,6 \mathrm{~L} \mathrm{~h}^{-1}$, mostrando a vantagem desse método em relação aos demais (convencional e reduzido). SCHOROCK et al. (1985), em sistema de plantio direto, encontraram 
consumo de combustível de 11,34 $\mathrm{L} \mathrm{h}^{-1}$. MAHL (2006) encontrou, em média, $12 \%$ de aumento no consumo horário de combustível para cada $\mathrm{km} \mathrm{h}^{-1}$ de aumento na velocidade de deslocamento, na operação de semeadura; neste trabalho, ocorreu aumento de $6,8 \%$, de 4,0 para $5,0 \mathrm{~km} \mathrm{~h}^{-1}$, e $11,5 \%$ de 5,0 para $6,0 \mathrm{~km} \mathrm{~h}^{-1}$.

Com relação ao consumo operacional de combustível, que foi influenciado pela velocidade de deslocamento, se relacionar com a capacidade de campo operacional, nota-se que ocorreu aumento de $16,1 \%$ e $22,0 \%$ da menor velocidade para a intermediária e desta para a maior, respectivamente, enquanto ocorreu diminuição do consumo operacional em 9,5\% e 9,3\%, respectivamente.

TABELA 3. Consumo horário e operacional de combustível e patinagem na operação de semeadura.

\begin{tabular}{|c|c|c|c|}
\hline Fatores & Consumo Horário $\left(\mathrm{L} \mathrm{h}^{-1}\right)$ & Consumo Operacional $\left(\mathrm{L} \mathrm{ha}^{-1}\right)$ & Patinagem $(\%)$ \\
\hline \multicolumn{4}{|l|}{ Manejos } \\
\hline Rolo-faca & $14,3 \mathrm{a}$ & $8,1 \mathrm{a}$ & 10,1 \\
\hline Triturador de palhas & $14,2 \mathrm{a}$ & $8,1 \mathrm{a}$ & 8,8 \\
\hline Roçadora & $14,3 \mathrm{a}$ & $8,1 \mathrm{a}$ & 7,7 \\
\hline \multicolumn{4}{|l|}{ Velocidades $\left(\mathrm{km} \mathrm{h}^{-1}\right)$} \\
\hline 4,0 & $13,2 \mathrm{c}$ & $8,8 \mathrm{a}$ & 10,1 \\
\hline 5,0 & $14,0 \mathrm{~b}$ & $8,1 \mathrm{~b}$ & 9,1 \\
\hline 6,0 & $15,7 \mathrm{a}$ & $7,4 \mathrm{c}$ & 7,0 \\
\hline \multicolumn{4}{|l|}{ Teste de F } \\
\hline Manejos (M) & $0,1^{\mathrm{NS}}$ & $0,2^{\mathrm{NS}}$ & 1,0 \\
\hline Velocidade (V) & $103,9 * *$ & $104,6 * *$ & 9,6 \\
\hline $\mathrm{Mx} \mathrm{V}$ & $0,6^{\mathrm{NS}}$ & $0,5^{\mathrm{NS}}$ & 0,8 \\
\hline C.V.(\%) - Manejos & 4,2 & 5,8 & 73,3 \\
\hline C.V. (\%) - Velocidade & 4,3 & 4,3 & 40,2 \\
\hline
\end{tabular}

\section{CONCLUSÕES}

O aumento da velocidade provocou a diminuição da força de tração e o aumento da capacidade de campo operacional e potência na barra.

O consumo horário de combustível aumentou com a velocidade, enquanto o consumo operacional diminuiu.

O desempenho da semeadora não foi influenciado pelos manejos efetuados na cobertura vegetal de inverno.

\section{REFERÊNCIAS}

ASAE. AMERICAM SOCIETY OF AGRICULTURAL ENGINEERS. Agricultural machinery management data. In: ASAE standards 1996: standards engineering practices data. St. Joseph, 1996. p.332-39.(ASAE D-497.2).

BOLLER, W.; KLEIN, V.A.; DALLMEYER, A.U.; SCHONS, P. Força de tração e potência para operar com uma semeadora-adubadora de precisão em solo sob preparo reduzido. In:

CONGRESSO BRASILEIRO DE ENGENHARIA AGRÍCOLA, 29., 1991, Londrina. Anais...

Londrina: SBEA/Instituto Agronômico do Paraná, 1992. p.1.111-23.

EMBRAPA. EMPRESA BRASILEIRA DE PESQUISA AGROPECUÁRIA. Sistema brasileiro de classificação de solos. Brasília,1999. 412 p. 
FURLANI, C.E.A. Sistema de manejo e rotação de culturas de cobertura em plantio direto de soja e milho. 2005. 99 f. Tese (Livre-Docência) - Faculdade de Ciências Agrárias e Veterinárias, Universidade Estadual Paulista, Jaboticabal, 2005.

JUSTINO, A. Desempenho do mecanismo dosador de disco perfurado horizontal na distribuição de sementes de milho (Zea mays L.), em sistema plantio direto. 1998. $104 \mathrm{f}$. Tese (Doutorado em Energia na Agricultura) - Faculdade de Ciências Agronômicas, Universidade Estadual Paulista, Botucatu, 1998.

KLEIN, V.A.; SIOTA, T.A.; ANESI, A.L.; BARBOSA, R. Efeitos da velocidade na semeadura direta de soja. Engenharia Agrícola, Jaboticabal, v.22, n.1, p.75-82, 2002.

LOPES, A.; FURLANI, C.E.A.; SILVA, R.P. Desenvolvimento de um protótipo para medição do consumo de combustível em tratores. Revista Brasileira de Agroinformática, Lavras, v.5, n.1, p.2431, 2003.

MAHL, D. Desempenho de semeadora-adubadora de milho (Zea mays L.) em sistema de plantio direto. 2002. 160 f. Dissertação (Mestrado em Energia na Agricultura) - Faculdade de Ciências Agronômicas, Universidade Estadual Paulista, Botucatu, 2002.

MAHL, D.; GAMERO, C.A.; BENEZ, S.H.; FURLANI, C.E.A.; SILVA, A.R.B. Demanda energética e eficiência na distribuição de sementes de milho sob variação de velocidade e condição do solo. Engenharia Agrícola, Jaboticabal, v.24, n.1, p.150-7, 2004.

MAHL, D. Desempenho de semeadora em função de mecanismo de corte, velocidade e solos, no sistema plantio direto do milho. 2006. 143 f. Tese (Doutorado em Energia na Agricultura) Faculdade de Ciências Agronômicas, Universidade Estadual Paulista, 2006.

MARQUES, J.P.; PONTES, J.R.V.; BENEZ, S.H. Desempenho de uma semeadora-adubadora de precisão na semeadura de milho (Zea mays L.) em preparo convencional de solo e em semeadura direta. In: CONGRESSO BRASILEIRO DE ENGENHARIA AGRÍCOLA, 28., 1999, Pelotas. Anais... Pelotas: Sociedade Brasileira de Engenharia Agrícola, 1999. 1 CD-ROM.

MIALHE, L.G. Ensaio e certificação de tratores. In: MIALHE, L.G. Máquinas agrícolas: ensaio e certificação. Piracicaba: Fundação de Estudos Agrários “Luiz de Queiroz”, 1996. cap. 8, p.385462.

NAGAOKA, A.K.; NOMURA, R.H.C. Tratores: semeadura. Cultivar Máquinas, Pelotas, n.18, p.24-6, janeiro/abril, 2003.

PIMENTEL GOMES, F. A estatística moderna na agropecuária. Piracicaba: Associação Brasileira para Pesquisa da Potassa e do Fosfato, 1987. 162 p.

SCHROCK, M.D.; KRAMER, S.J.; CLARK, S.J. Fuel requirements for field operations in Kansas. Transaction of the ASAE, St. Joseph, v.28, n.3, p.669-74, 1985.

SILVA, S.L. Avaliação de semeadoras para plantio direto: demanda energética, distribuição longitudinal e profundidade de deposição de sementes em diferentes velocidades de deslocamento. 2000. 123 f. Tese (Doutorado em Energia na Agricultura) - Faculdade de Ciências Agronômicas, Universidade Estadual Paulista, Botucatu, 2000.

SIQUEIRA, R.; BOLLER, W.; GAMERO, C.A. Capacidade de trabalho e consumo de combustível na trituração de três coberturas vegetais. In: CONGRESSO BRASILEIRO DE ENGENHARIA AGRÍCOLA, 26., 1997, Campina Grande. Anais... Campina Grande: Sociedade Brasileira de Engenharia Agrícola, 1997. 1 CD-ROM. 